\title{
SPIN DOWN OF BOUSSINESQ FLUID IN THE CIRCULAR \\ CYLINDER, AS A SIMULATION OF THE SOLAR \\ SPIN-DOWN PROCEDURE
}

\author{
TAKEO SAKURAI \\ Dept. of Aeronautical Engineering, Kyoto University, Kyoto, Japan
}

\begin{abstract}
As the simplest model to simulate the solar spin down procedure from the hydrodynamical viewpoint, a spin down problem with a simple geometrical configuration within the frame work of the Boussinesq approximation is investigated. A new proposal of the origin of the solar differential rotation is made on the basis of the calculated asymptotic distribution of the angular velocity. A comment on the present day solar spin down controversy is also made.
\end{abstract}

\section{Introduction}

In Hoyle's theory of the origin of the solar system and several other studies of stellar rotation (Hoyle, 1960; Lüst and Schlüter, 1955; Dicke, 1964; Brandt, 1966), it is shown that the solar angular momentum is lost by the magnetohydrodynamic interaction with the interplanetary medium, and that the primeval solar angular momentum rotating once a day is lost in the time duration of the solar age or of an order of magnitude larger.

What effect does this have on the rotation in the sun? This question is fundamental not only in itself but also as the mediator of the solar spin down controversy. Dicke (1964) made an importan suggestion that the solar interior rotates once a day in spite of the observational evidence that the solar surface rotates once in 27 days. Howard et al. (1967) opposed Dicke on the basis of the spin down procedure in the solar interior; that is the angular momentum rearrangement by the meridional circulation induced by the pumping mechanism of the Ekman's boundary layer. Thereafter, several authors discussed the validity of Dicke's hypothesis (Fricke and Kippenhahn; Dicke 1967; Bretherton and Spiegel, 1968). However, no conclusive result has yet been obtained.

As the simplest model to simulate the unsteady rotational motion of the sun under the influence of the angular momentum loss, the following is investigated in this paper: Viscous heat conducting compressible fluid rotates rigidly with the containing circular cylinder rotating uniformly around the vertical axis of symmetry under the influence of the constant gravitational force and the stable distribution of the temperature. The angular velocity of the cylinder is changed abruptly at a certain instant of time while the horizontal wall of the cylinder is made to keep to the original temperature and the vertical wall is made to keep to the original temperature or to be held thermally insulated. Our problem is to study the response of the fluid to the above abrupt change within the time scale of the incompressible spin down time and within the frame work of the Boussinesq approximation. The asymptotic distribution of the angular velocity and of the temperature especially are studied in detail. 
The overall character of the response is divided into three steps according to the respective time scales: Within the time scale of the rotational period (the reciprocal of the angular velocity), Ekman's boundary layers are established on the horizontal walls and the meridional circulation is generated by the pumping mechanism of the Ekman's boundary layer. Within the time scale of the spin down time (the geometrical mean of the rotational period and the following diffusion time), the angular momentum is transferred by the meridional current and the main bulk of the inviscid region is spun down. On the other hand, the above spin down procedure is partially quenched by the effect of the stable stratification, and there appears a non-uniformity in the angular velocity distribution. Finally, within the time scale of the diffusion time (the square of the representative length divided by the kinematic viscosity), the above non-uniformity is relaxed by the effect of the viscous diffusion to realize the final state of the rigid rotation corresponding to the disturbed angular velocity of the containing cylinder.

The rotational period of the sun is about a day or 27 days (according to Dicke's hypothesis or to the observational evidence), while the spin down time is estimated to be of the order of $10^{5-7}$ years and the diffusion time to be of the order of $10^{11}$ years. The solar age, on the other hand, is estimated to be of the order of $10^{9}$ years. Thus, the present status of the solar rotation is understood as the asymptotic state within the time scale of the spin down time.

The above problem is a source of controversy also in the field of hydrodynamics. Holton (1965) investigated the spin down of rotating stratified fluid in the circular cylinder by a heuristic treatment and showed the existence of the spin down procedure similar but not identical to that in the incompressible fluid. Pedlosky (1967) re-examined the same problem from the viewpoint of complementing Holton's treatment and gave a clearer formulation. Pedlosky's conclusion for the case with a thermally insulated vertical wall is that the inviscid region is spun down by a strictly diffusive process within the time scale of the diffusion time, which conclusion is completely at variance with that of Holton. Holton and Stone (1968) examined Pedlosky's solution, pointed out the inconsistency in the solution, and suggested a further study of the same problem.

Recently, Sakurai (1969a, b) investigated the above problem in detail and resolved the Holton-Pedlosky controversy analytically. He did not, however, make a detailed numerical study of the asymptotic distribution of the angular velocity and the temperature. Thus, the present study is to be taken as the extension of his previous investigations. Since the analytical treatment is completely the same as that in his previous investigations, the mathematical detail is omitted except that which is essential to understand the underlying mathematics.

\section{Basic Equations}

The basic equations governing the axisymmetric motion of viscous heat conducting compressible fluid within the frame work of the Boussinesq approximation written in 
cylindrical coordinates rotating with the angular velocity $\Omega$ about the vertical axis of symmetry are:

$$
\begin{aligned}
& \frac{\partial q_{r}}{\partial t}-2 q_{\theta}=-\frac{\partial p}{\partial r}+E \mathscr{L} q_{r}, \\
& \frac{\partial q_{\theta}}{\partial t}+2 q_{r}=E \mathscr{L} q_{\theta}, \\
& \frac{\partial q_{z}}{\partial t}=-\frac{\partial p}{\partial z}+T+E \Delta q_{z}, \\
& \sigma\left(\frac{\partial T}{\partial t}+S q_{z}\right)=E \Delta T, \\
& 0=\frac{1}{r} \frac{\partial}{\partial r}\left(r q_{r}\right)+\frac{\partial q_{z}}{\partial z}, \\
& E=\frac{v}{L^{2} \Omega}, \quad S=\frac{\alpha g\left(\bar{T}_{1}-\bar{T}_{0}\right)}{L \Omega^{2}}, \quad \sigma=\frac{v}{\kappa} \\
& \mathscr{L}=\Delta-\frac{1}{r^{2}}, \quad \Delta=\frac{\partial^{2}}{\partial r^{2}}+\frac{1}{r} \frac{\partial}{\partial r}+\frac{\partial^{2}}{\partial z^{2}},
\end{aligned}
$$

where

$$
\begin{aligned}
& (\bar{r}, 0, \bar{z})=(L r, \theta, L z), \quad \bar{t}=\frac{t}{\Omega}, \\
& \mathbf{q}=\varepsilon \Omega L \mathbf{q}, \quad \bar{p}=\bar{p}_{S}+\varepsilon \Omega^{2} L^{2} \bar{\varrho}_{S} p, \quad \bar{T}=\bar{T}_{S}+\varepsilon \frac{\Omega^{2} L}{\alpha g} T, \\
& \bar{\varrho}_{S}=\bar{\varrho}_{0}\left\{1-\frac{\alpha\left(\bar{T}_{1}-\bar{T}_{0}\right)}{L} \bar{z}\right\}, \bar{p}_{S}=\bar{p}_{0}-\int_{0}^{\bar{z}} g \bar{\varrho}_{S} \mathrm{~d} \bar{z}, \quad \bar{T}_{S}=\bar{T}_{0}+\frac{\bar{T}_{1}-\bar{T}_{0}}{L} \bar{z},
\end{aligned}
$$

and $\left(q_{r}, q_{\theta}, q_{z}\right), p, \varrho, T,(r, \theta, z), t, v, \kappa, \alpha, g, L$ and $\varepsilon$ are the velocity, the pressure, the density, the temperature, the position vector, the time, the viscosity, the thermal conductivity, the coefficient of thermal expansion, the gravitational acceleration, the height of the cylinder and the parameter corresponding to the small deviation from the state of rotating equilibrium, and suffixes $S, 1$ and 0 and bars on letters refer to the state of rotating equilibrium, the upper and lower bottom of the cylinder and to the original physical quantity with dimension, respectively. In the derivation of the above linear equations from the full basic equations, terms of the order of $\varepsilon^{2}$ and $\Omega^{2} L / g$ are neglected as is exemplified in the steady flow (Barcilon and Pedlosky, 1967). $E$ is assumed to be very small while $S$ and $\sigma$ are taken to be of the order of 1 . Hence, our investigation falls within the parameter range of compressibility dominant cases, in which the Ekman's pumping mechanism does not work, according to Barcilon and Pedlosky's classification based on the steady solution. 
The introduction of the stream function of the meridional current leads to the following:

$$
\begin{aligned}
E \tilde{\mathscr{L}} & \left\{E^{1 / 2} \tilde{\mathscr{L}}-\frac{\partial}{\partial \tilde{t}}\right\} \tilde{\psi}+2 \frac{\partial \tilde{q}_{\theta}}{\partial \tilde{z}}=\frac{\partial \tilde{T}}{\partial \tilde{r}}, \\
& \left\{E^{1 / 2} \tilde{\mathscr{L}}-\frac{\partial}{\partial \tilde{t}}\right\} \tilde{q}_{\theta}-2 \frac{\partial \tilde{\psi}}{\partial \tilde{z}}=0, \\
& \left\{E^{1 / 2} \tilde{\Delta}-\sigma \frac{\partial}{\partial \tau}\right\} \tilde{T}+\frac{\sigma S}{\tilde{r}} \frac{\partial}{\partial \tilde{r}}(\tilde{r} \tilde{\psi})=0,
\end{aligned}
$$

where

$$
\begin{aligned}
& q_{r}=\frac{\partial \psi}{\partial z}, \quad q_{z}=-\frac{1}{r} \frac{\partial}{\partial r}(r \psi), \\
& r=\tilde{r}, \quad z=\tilde{z}, \quad t=E^{-1 / 2} \tilde{t}, \\
& \psi=E^{1 / 2} \tilde{\psi}, \quad q_{\theta}=\tilde{q}_{\theta}, \quad T=\tilde{T} .
\end{aligned}
$$

The transformation (15) corresponds to the time duration in which our investigation is aimed. The quantities with tildes are assumed to be of the order of 1 . Hence, (15) implies the existence of the meridional current with the same order of magnitude as that in the incompressible flow, showing the essential difference between the steady and the unsteady motion.

The initial conditions are obtained by the expression that the fluid is in rotating equilibrium until a certain instant:

$$
\tilde{\psi}=\tilde{q}_{\theta}=\tilde{T}=0, \quad \text { for } \quad 0 \leqslant \tilde{z} \leqslant 1, \quad 0 \leqslant \tilde{r} \leqslant r_{0}, \quad \tilde{t}<0
$$

where $r_{0}$ is the ratio of the radius to the height of the cylinder.

The boundary conditions are obtained by the expression that the angular velocity of the cylinder is abruptly changed after the above instant while the horizontal wall of the cylinder is made to keep to the original temperature and the vertical wall is (A) to keep to the original temperature or (B) to be held thermally insulated:

$$
\begin{aligned}
& \tilde{\psi}=\partial \tilde{\psi} / \partial \tilde{z}=\tilde{T}=0, \quad \tilde{q}_{\theta}=\omega \tilde{r}, \quad \text { for } \\
& \tilde{z}=0 \text { or } 1, \quad 0 \leqslant \tilde{r} \leqslant r_{0}, \quad \tilde{t}>0
\end{aligned}
$$

(A) $\tilde{\psi}=\partial \tilde{\psi} / \partial \tilde{r}=\tilde{T}=0, \quad \tilde{q}_{\theta}=\omega r_{0}$,

(B) $\tilde{\psi}=\partial \tilde{\psi} / \partial \tilde{r}=\partial \tilde{T} / \partial \tilde{r}=0, \quad \tilde{q}_{\theta}=\omega r_{0}$.

for

$$
0 \leqslant \tilde{z} \leqslant 1, \quad \tilde{r}=r_{0}, \quad \tilde{t}>0
$$

\section{Similar Solution}

Before going ahead directly into the examination of the numerical results of the asymptotic distributions calculated on the basis of the solution of (11) to (13) under the initial and boundary conditions (16) to (19), it is interesting to note that there is 
the following similar solution for the limiting case with the infinitely large radius of the cylinder:

$$
\psi=r \phi(z, t), \quad q_{\theta}=r v(z, t), \quad T=T(z, t)
$$

where tildes over letters are omitted for the sake of simplicity.

The substitution of (20) into (11) to (13) leads to the following:

$$
\begin{gathered}
E \frac{\partial^{2}}{\partial z^{2}}\left\{E^{1 / 2} \frac{\partial^{2}}{\partial z^{2}}-\frac{\partial}{\partial t}\right\} \phi+2 \frac{\partial v}{\partial z}=0, \\
\left\{E^{1 / 2} \frac{\partial^{2}}{\partial z^{2}}-\frac{\partial}{\partial t}\right\} v-2 \frac{\partial \phi}{\partial z}=0, \\
\left\{E^{1 / 2} \frac{\partial^{2}}{\partial z^{2}}-\sigma \frac{\partial}{\partial t}\right\} T+2 \sigma S \phi=0 .
\end{gathered}
$$

Equations (21), (22) and the corresponding initial and boundary conditions are nothing but those treated in the incompressible case (Greenspan and Howard, 1963). It is concluded, therefore, that the state of rigid rotation is established within the time duration of the incompressible spin down time without regard to the stratification in the present limiting case. The important point to be stressed here is that this limiting case is not singly isolated from other cases but proceeds continuously as $r_{0}$ is made larger and larger. Another important phenomenon which the above similar solution implies is that the thickness of the thermal boundary layer is of the order of $E^{1 / 4}$, unlike the velocity boundary layer, as is seen by (23). The meridional current driven by the Ekman's boundary layer modifies not only the velocity but also the temperature outside the boundary layer. The matching of this convectively modified temperature with the wall temperature is achieved via the thermal boundary layer of the above thickness where thermal conduction is predominant. The appearance of this thermal boundary layer on the horizontal surface is characteristic of the transient variation within the time duration of the order of the incompressible spin down time and has no analogue in the steady flow problem. It is naturally expected that, for the case with finite $r_{0}$, the intermediate layer of the thickness of the order of $E^{1 / 4}$ coexists together with the inner layer of the thickness of the order of $E^{1 / 2}$ also along the vertical wall.

Therefore, the flow field in the general case is divided into five regions: In the inviscid region, the Coriolis force, the buoyancy force and the pressure dominate in the flow field while the temperature is governed by the convection. In the horizontal inner layer, that is in the Ekman's boundary layer, the Coriolis force is balanced by the shearing stress in the flow field, and the peripheral velocity is coupled with the meridional velocity. In the horizontal intermediate layer, the non-diffusive temperature in the inviscid region is relaxed into the wall temperature by thermal conduction. In the vertical inner layer, the buoyancy is balanced by the shearing stress of the meridional current while thermal convection is balanced by thermal conduction, and 
the meridional current is coupled with the temperature. In the vertical intermediate layer, the inviscid peripheral velocity is relaxed into the wall velocity by the viscous diffusion.

The mathematical formulation of the above features was made by Sakurai (1969a, b) who gave a detailed analytical discussion of the flow field. Therefore, we do not get into trouble with the mathematical complexity except noting the following: Since the boundary layers are very thin within our time scale, the inviscid region consists of the main bulk of the flow field, and the mathematical function of the former is to provide us with the boundary conditions to be satisfied by the latter. Our problem reduces, thus, to the solution of the initial boundary value problem with respect to the inviscid flow. The solution is obtained by the expansion with respect to the fundamental solutions which, in turn, are obtained by the method of the separation of variables. The convergence of the asymptotic form of this expansion becomes poor for the temperature in the neighborhood of $r / r_{0}=0$ and 1 . This is the reason why we get some extraordinary behavior in Figures 3, 4, 7 and 8 in the same region.

Finally, the meridional circulation dies away in the asymptotic state, and the asymptotic distribution of the angular velocity and the temperature is governed by a single parameter $\sqrt{ } S / r_{0}$. This leads to the equivalence of the case with infinite radius of the cylinder to that with vanishing stratification. Thus, our solution certainly includes the former as a special case.

\section{Results and Discussion}

The numerical calculation of the asymptotic distribution of the angular velocity and the temperature based on Sakurai's analytical formulation was performed on TOSBAC3400 electronic computer of the Research Institute of Mathematical Science of Kyoto University, and the results are shown in Figures 1 to 8 . These figures clearly show the situations where the effect of the meridional current is quenched, and thus,

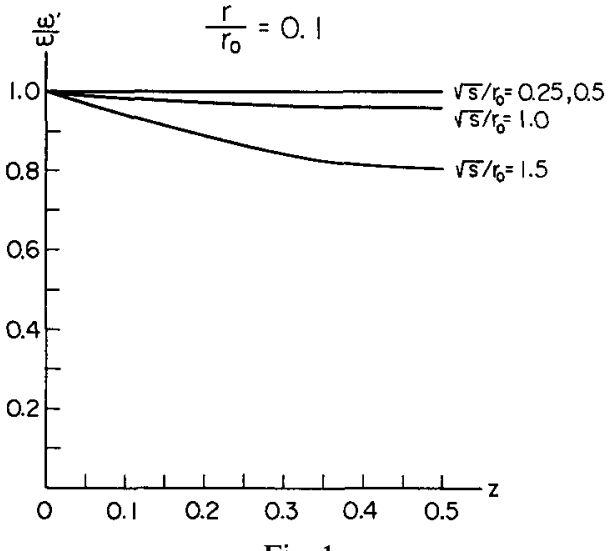

Fig. 1.

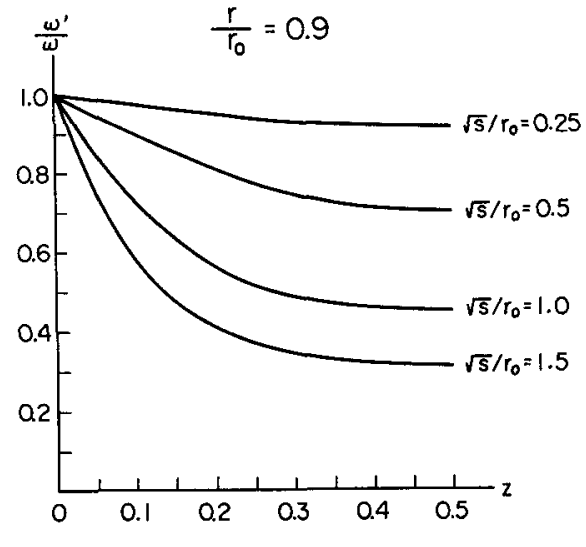

Fig. 2.

Figs. 1 and 2. Asymptotic distributions of the perturbation angular velocity as functions of the height with a radius and stratification numbers as parameters. Case $\mathrm{A}$. 


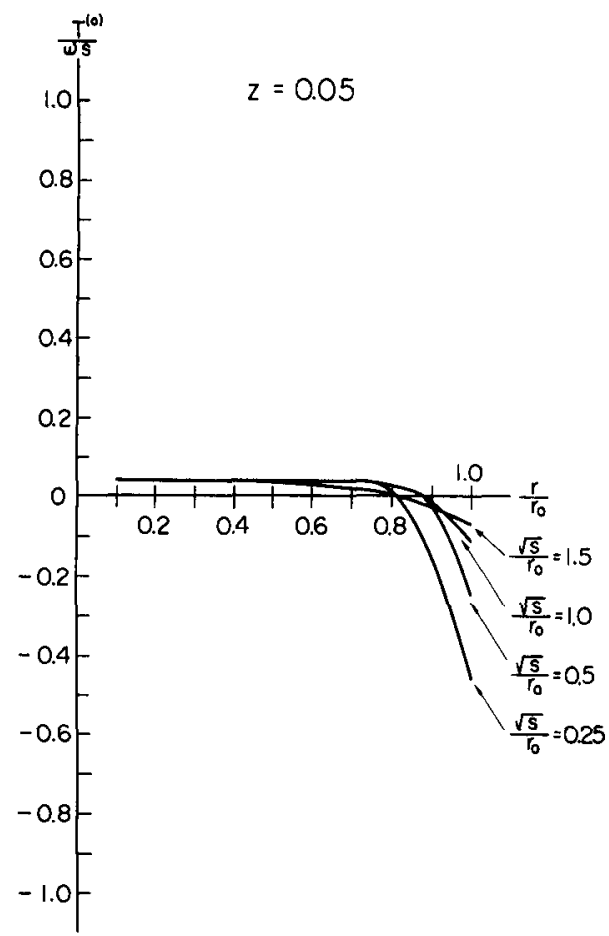

Fig. 3.

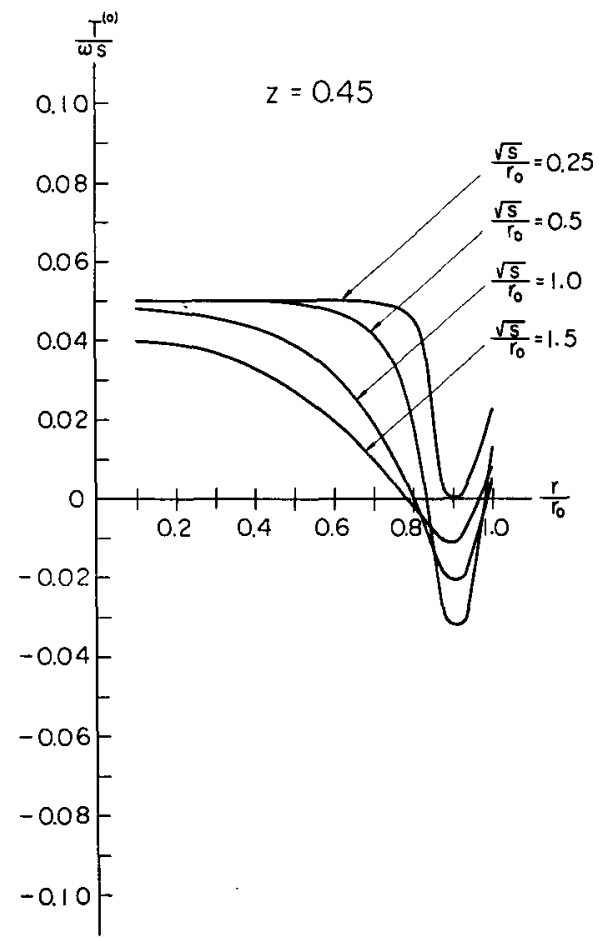

Fig. 4.

Figs. 3 and 4. Asymptotic distributions of the perturbation temperature as functions of the radius with a height and stratification numbers as parameters. Case $\mathbf{A}$.

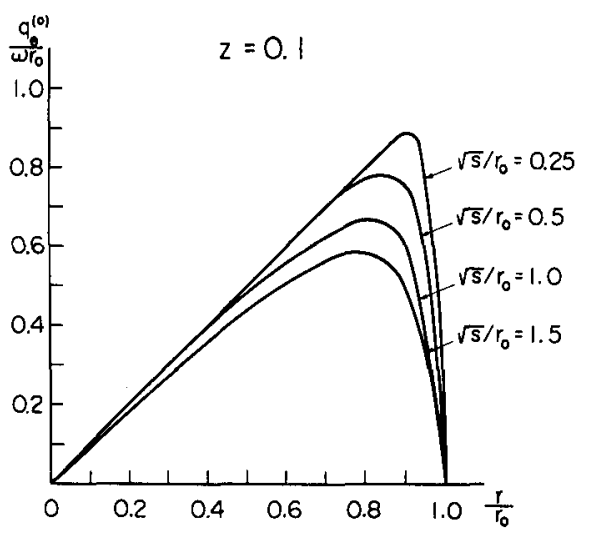

Fig. 5.

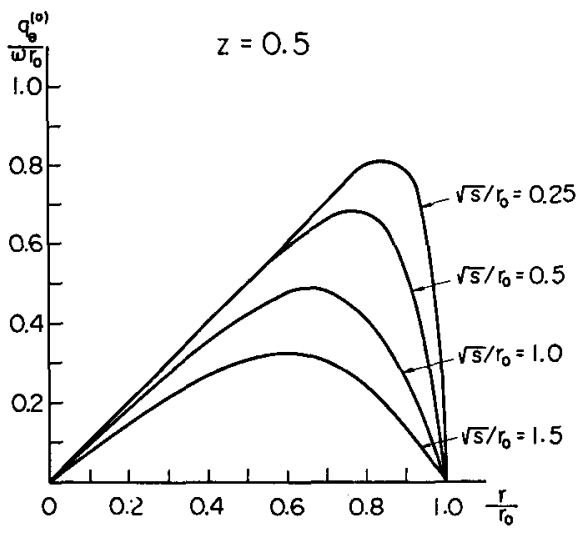

Fig. 6.

Figs. 5 and 6. Asymptotic distributions of the perturbation peripheral velocity as functions of the radius with a height and stratification numbers as parameters. Case $B$. 


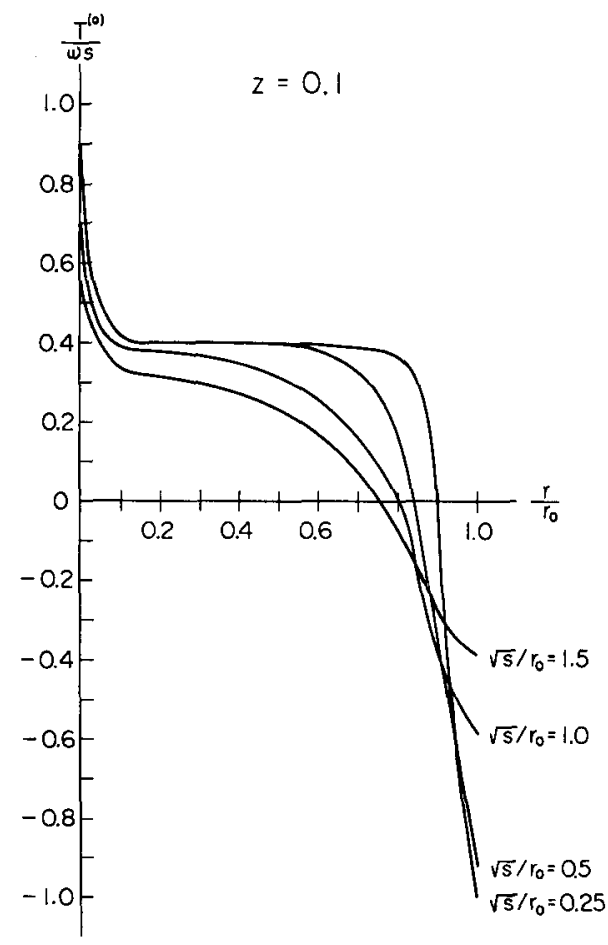

Fig. 7.

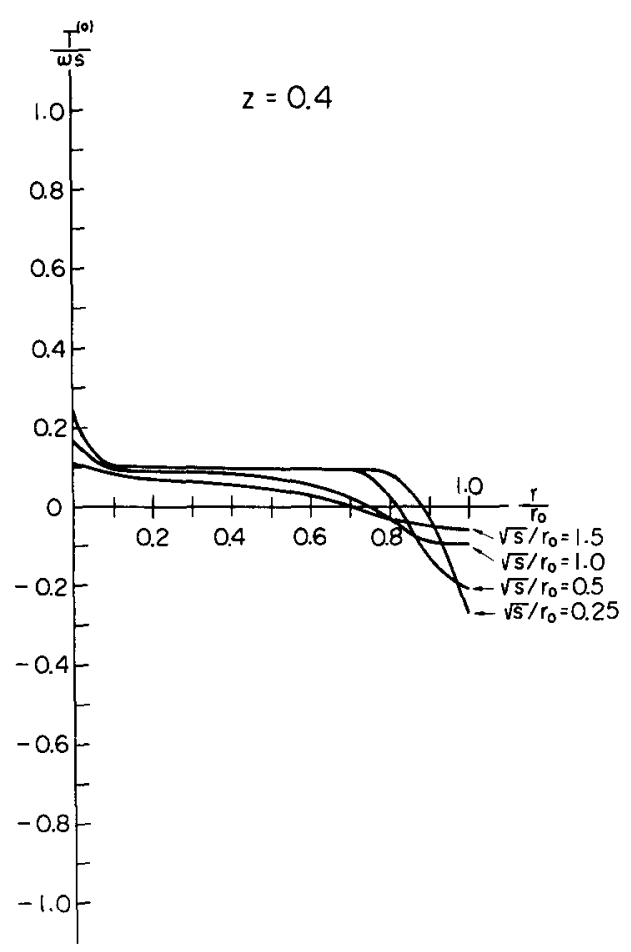

Fig. 8.

Figs. 7 and 8. Asymptotic distributions of the perturbation temperature as functions of the radius with a height and stratification numbers as parameters. Case B.

the non-uniformity in the flow field is augmented by the stable stratification. The most interesting feature, however, is that our asymptotic angular velocity distribution in case A shows the equatorial acceleration to be similar to that on the solar surface: the absolute value of the perturbation peripheral velocity is the smallest in the equatorial region on the constant $r$-plane, corresponding to the equatorial acceleration in the spin down case (Figures 1 and 2). This may be due to the nonuniformity in the conflict between the pumping mechanism of the Ekman's boundary layer and the quenching action of the stable stratification with respect to the driving of the meridional circulation and hence to the transfer of the angular momentum. The mechanism is explained more intuitively as follows: The horizontal surface of our problem is taken to be equipped with many pumps which have approximately the same power and are connected to bad hoses (Figure 9). These hoses are partially choked by the effect of the stable stratification with respect to the angular momentum transport, and the effect of choking becomes larger and larger as the equatorial region is approached. Then, it is evident that we have a smaller amount of angular momentum transport in the equatorial region than elsewhere, to obtain the equatorial acceleration in the spin down case. It is noted that the qualitative nature of this procedure is quite general without regard to the geometrical configuration and the origin 
of the pumping mechanism. In effect, the above mechanism does work in the solar interior where we have a stronger effect of the stratification and a more powerful pumping mechanism by Bretherton and Spiegel (1968). Thus, our result is taken to suggest a possible explanation of the origin of the solar differential rotation.

The details of the procedure are, of course, influenced by (1) the nature of the mechanical interaction between the hydrogen convection layer and the inner stable region, (2) the latitudinal distribution of the magnetohydrodynamical breaking torque
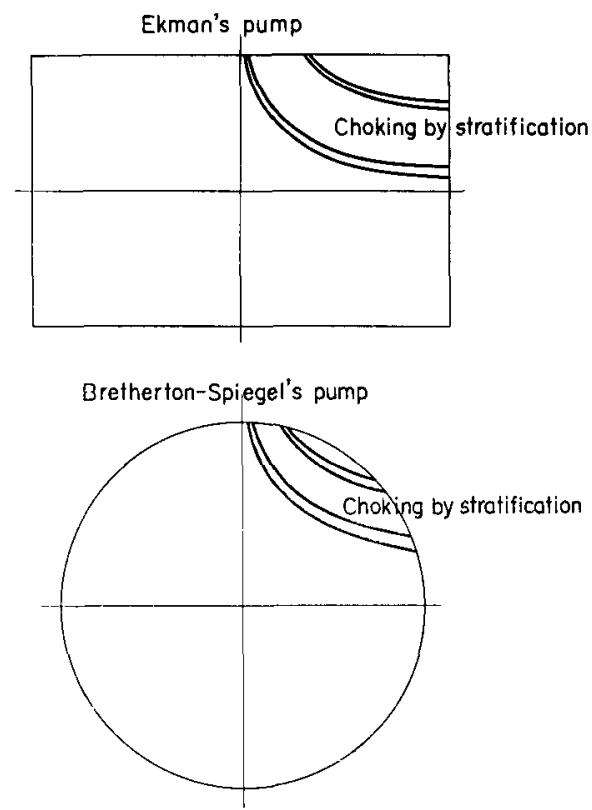

Fig. 9. Intuitive explanation of the origin of equatorial acceleration.

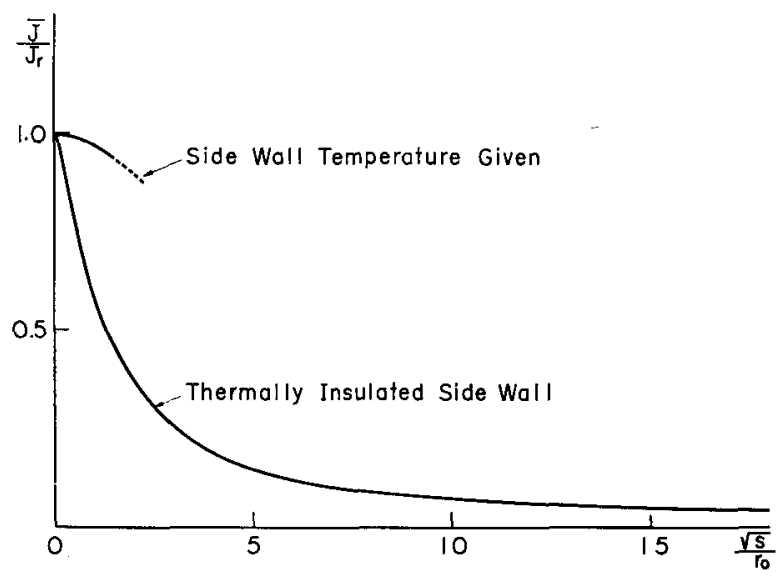

Fig. 10. Asymptotic amounts of the transferred angular momentum as functions of the stratification number. 
on the surface of the sun, (3) the solar magnetic field, and so on. However, the general features may remain the same.

The explanation based on the non-isotropy of the eddy viscosity in the hydrogen convection layer (Sakurai, 1966) has the fatal defect that the estimated order of magnitude of the meridional current on the solar surface is much larger than that of the observed one. The present explanation has no such defect, since the meridional circulation dies away in the asymptotic state. Recently, Kato and Nakagawa (1968, 1969) showed that the Rossby wave is excited in the differentially rotating solar photosphere and that the thus excited Rossby wave induces such an angular momentum transfer as to sustain the equatorial acceleration. Our mechanism of the origin of the solar differential rotation may trigger this effect of the Rossby wave.

The non-uniformity of the angular velocity distribution is also shown by the asymptotic amount of the transferred angular momentum $\bar{J} / \bar{J}_{r}$, as is shown in Figure 10, where

$$
J=2 \pi \int_{0}^{L} \int_{0}^{L r_{0}} \bar{r}^{2} \bar{\varrho} \bar{q}_{\theta} \mathrm{d} \bar{r} \mathrm{~d} \bar{z},
$$

and the suffix $r$ refers to the rigid body rotation. It is interesting that the amount is quite remarkable in the case (A) even for a moderate value of $S$. It is also interesting that the amount depends critically on the thermal condition on the vertical wall. This is a very important point to be careful about from the viewpoint of constructing a more concrete model of the solar spin down phenomenon.

Let us apply our result to the solar interior, even though it is not permitted because of the limitations on the Boussinesq approximation. The term $\alpha\left(\bar{T}_{1}-\bar{T}_{0}\right)$ in (6) is taken to be of the order of 100 since it represents the fractional order of the density variation. Then, the main parameter $\sqrt{ } S / r_{0}$ is estimated to be of the order of 100 corresponding to the rate of 1 rotation per day. The asymptotic amount of the transferred angular momentum is very small as is seen in Figure 10, and we cannot expect the effective spin down of the solar interior, which result seems to ascertain Dicke's hypothesis.

However, we are more interested in the fact that our results clearly show the occurrence of the spin down within our time duration. We want, therefore, to be prudent not to give any judgement about the solar spin down controversy. In effect, Bretherton and Spiegel (1968) proposed that a slow circulation in the convection zone induced by the gradual reduction in the rotation of the solar surface layer pumps fluid into the solar interior and gives rise to spin down currents which are much more intense than those resulting from a solar Ekman layer. Thus, all we can say is that the only way to resolve the solar spin down controversy is to investigate the unsteady rotation of the model solar interior.

\section{Acknowledgement}

The author wishes to express his cordial thanks to Mr. S. Yamamoto for his assistance in performing numerical calculations. 


\section{References}

Barcilon, V. and Pedlosky, J.: 1967, J. Fluid Mech. 29, 1.

Brandt, J. C.: 1966, Astrophys. J. 144, 1221.

Bretherton, F. P. and Spiegel, E. A.: 1968, Astrophys. J. 153, L77.

Dicke, R. H.: 1964, Nature 202, 432.

Dicke, R. H.: 1967, Astrophys. J. 149, L121.

Fricke, K. and Kippenhahn, R.: Publ. Univ. Sternwarte Göttingen.

Greenspan, H. P. and Howard, L. N.: 1963, J. Fluid Mech. 17, 385.

Holton, J. R.: 1965, J. Atmos. Sci. 22, 402.

Holton, J. R. and Stone, P. H.: 1968, J. Fluid Mech. 33, 127.

Howard, L. N., Moore, D. W., and Spiegel, E. A.: 1967, Nature 214, 1297.

Hoyle, F.: 1960, Quart. J. Roy. Astron. Soc. 1, 28.

Kato, S. and Nakagawa, Y.: 1968, Res. Note of H.A.O.

Kato, S. and Nakagawa, Y.: 1969, Res. Note of H.A.O.

Lüst, V. R. and Schlüter, A.: 1955, Z. Astrophys. 38, 190.

Pedlosky, J.: 1967, J. Fluid Mech. 28, 463.

Sakurai, T.: 1966, Publ. Astron. Soc. Japan 8, 174.

Sakurai, T.: 1969a, J. Phys. Soc. Japan 26, 840.

Sakurai, T.: 1969b, J. Fluid Mech. 37, 689.

\section{Discussion}

Roxburgh: This summer in Boulder I heard two colloquia, one by Clark and the other by someone else - both of whom claimed that spin down would not work in a strongly stratified fluid. Ekman pumping worked in a thin layer where the stratification was weak but did not penetrate the stable layers.

Sakurai: I have not read the papers you referenced. Therefore, I can say nothing about them. I ask you, however, to give due care to the possible existence of the solar spin down current driven by the Bretherton-Spiegel type pumping mechanism.

Dicke: If I understand Dr. Sakurai's calculation, it is based on the assumption that the angular velocity of the container is changed discontinuously. In this connection, the results of an experiment performed by my student $\mathrm{E}$. McDonald are significant. In his investigations of rotation of a density stratified fluid it was found that the density stratification stabilized the fluid against the formation of circulation currents and Ekman layers, but only if the rotation of the container was changed slowly and continuously. The physical reason for this is easily found. The state of rotation of the fluid determines the distribution of density, for rotation without circulation. Thus a sudden change in the rotation of the boundary will result in the wrong density distribution in the vicinity of the boundary. Thus purely rotational motion is impossible. McDonald found that a very small change in the angular velocity of the fluid container if suddenly applied would induce spin down of the fluid. Circulation currents would divide the fluid into two zones of uniform but different density. An Ekman layer would form at the container bottom and at the boundary between the two fluids. He also found that large differential rotations could be induced without spin down if the rotation of the fluid container was changed slowly. 